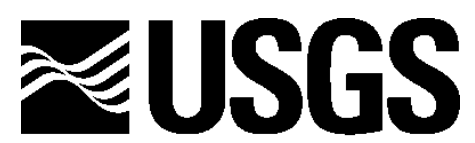

science for a changing world

\title{
An Interface for Simulating Radiative Transfer In and Around Volcanic Plumes with the Monte Carlo Radiative Transfer Model McArtim
}

By Christoph Kern

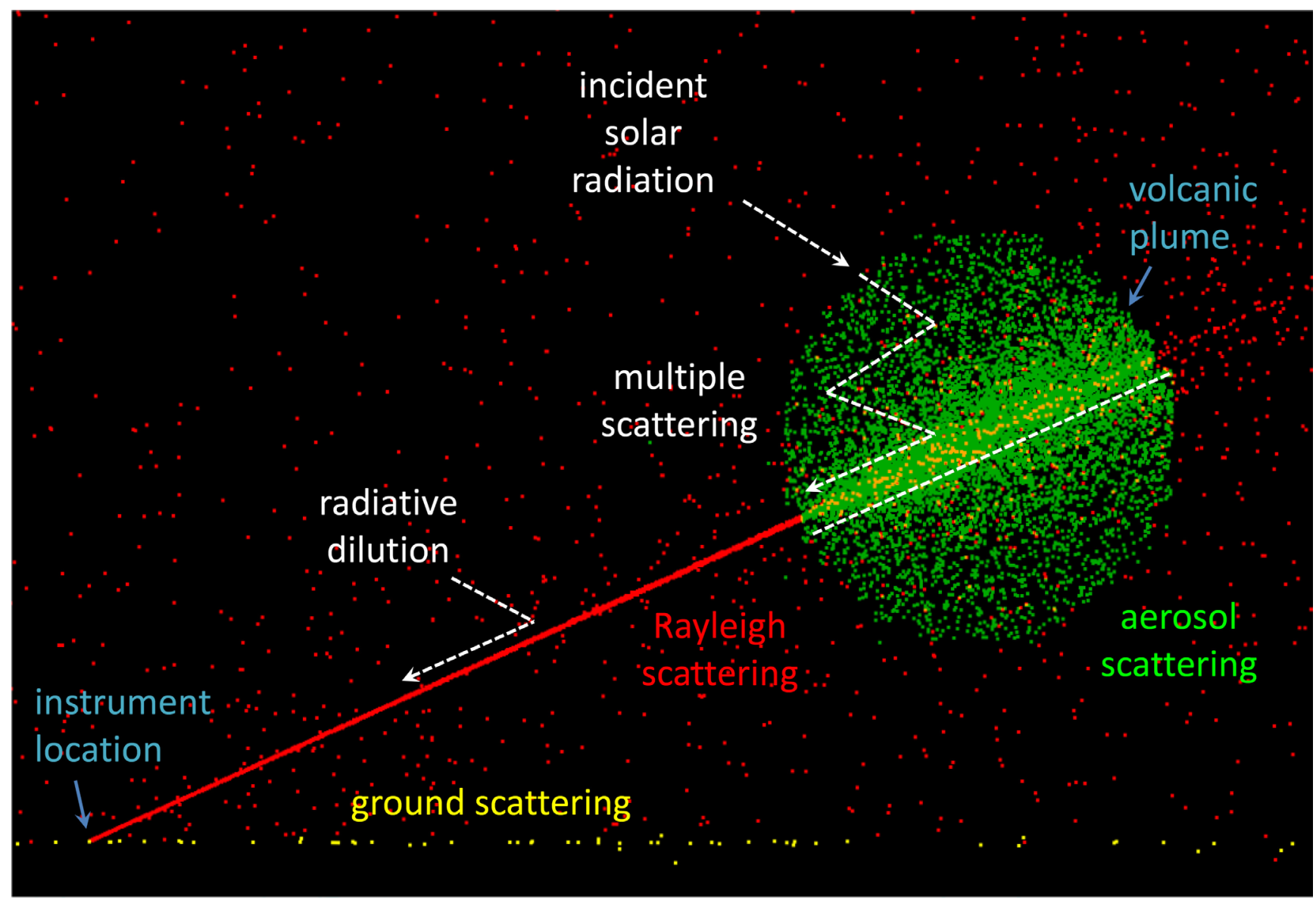

Open-File Report 2016-1045

U.S. Department of the Interior

U.S. Geological Survey 
Cover. Graphical representation of simulated radiative transfer in and around a volcanic plume. Red dots indicate Rayleigh scattering in the atmosphere, green dots indicate scattering on aerosols in the volcanic plume, and yellow dots indicate scattering on the ground. Radiation detected by an instrument (lower left corner) has taken a variety of different paths through or around the plume. 


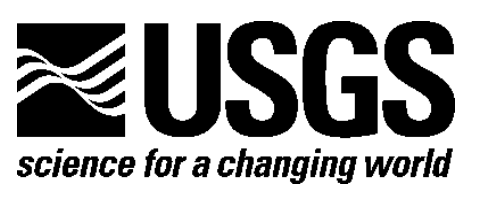

\section{An Interface for Simulating Radiative Transfer In and Around Volcanic Plumes with the Monte Carlo Radiative Transfer Model McArtim}

By Christoph Kern

Open-File Report 2016-1045

U.S. Department of the Interior

U.S. Geological Survey 


\section{U.S. Department of the Interior \\ SALLY JEWELL, Secretary}

\section{U.S. Geological Survey \\ Suzette M. Kimball, Director}

U.S. Geological Survey, Reston, Virginia: 2016

For more information on the USGS-the Federal source for science about the Earth,

its natural and living resources, natural hazards, and the environment-visit

http://www.usgs.gov/ or call 1-888-ASK-USGS (1-888-275-8747).

For an overview of USGS information products, including maps, imagery, and publications, visit http://www.usgs.gov/pubprod/.

Any use of trade, firm, or product names is for descriptive purposes only and does not imply endorsement by the U.S. Government.

Although this information product, for the most part, is in the public domain, it also may contain copyrighted materials as noted in the text. Permission to reproduce copyrighted items must be secured from the copyright owner.

Suggested citation:

Kern, Christoph, 2016, An interface for simulating radiative transfer in and around volcanic plumes with the Monte Carlo radiative transfer model McArtim: U.S. Geological Survey Open-File Report 2016-1045, 18 p., http://dx.doi.org/10.3133/ofr20161045.

ISSN 2331-1258 (online) 


\section{Contents}

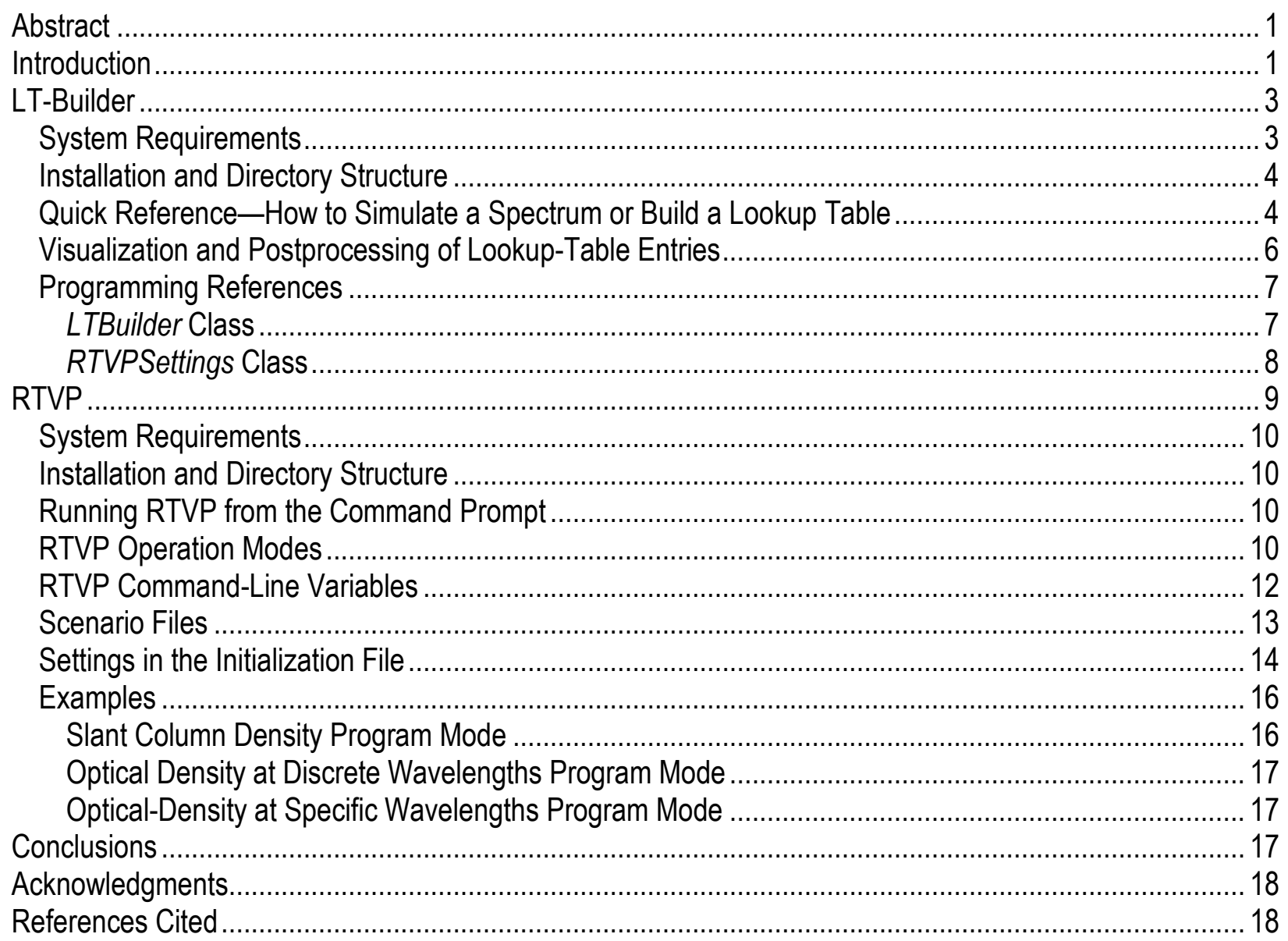

\section{Figures}

1. Diagram showing flow of information for simulating radiative transfer in and around volcanic plumes using the Lookup Table Builder (LT-Builder) and Radiative Transfer in Volcanic Plumes (RTVP) interfaces for the radiative transfer model (RTM) McArtim

2. Example MatLab script that can be used to generate a lookup table for use in sophisticated spectroscopic retrievals. This script (BuildLookupTableExample.m) is included in the software distribution of the Lookup Table Builder (LT-Builder) software.

\section{Tables}

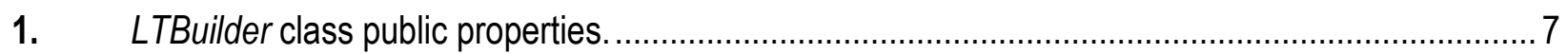

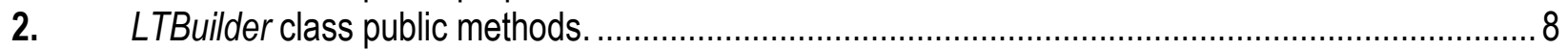

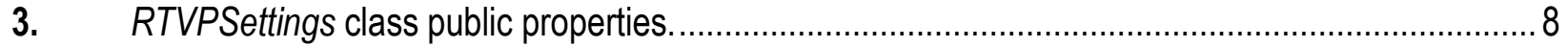

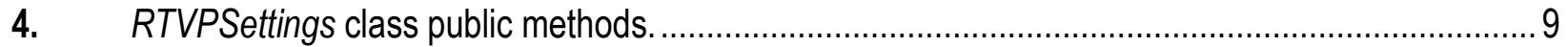

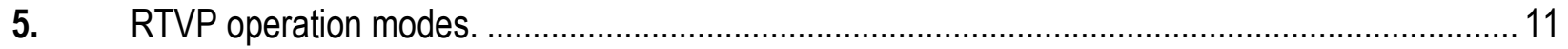

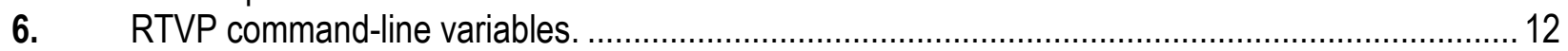

7. All settings configured in the RTVP_settings.ini initialization file (RTVP version 1.0.0)................ 14 


\section{Conversion Factors}

\begin{tabular}{|c|c|c|}
\hline Multiply & By & To obtain \\
\hline \multicolumn{3}{|c|}{ Length } \\
\hline centimeter $(\mathrm{cm})$ & 0.3937 & inch (in.) \\
\hline $\operatorname{meter}(\mathrm{m})$ & 3.281 & foot $(\mathrm{ft})$ \\
\hline kilometer $(\mathrm{km})$ & 0.6214 & mile (mi) \\
\hline \multicolumn{3}{|c|}{ Area } \\
\hline square centimeter $\left(\mathrm{cm}^{2}\right)$ & 0.001076 & square foot $\left(\mathrm{ft}^{2}\right)$ \\
\hline square centimeter $\left(\mathrm{cm}^{2}\right)$ & 0.1550 & square inch $\left(\mathrm{ft}^{2}\right)$ \\
\hline \multicolumn{3}{|c|}{ Volume } \\
\hline cubic centimeter $\left(\mathrm{cm}^{3}\right)$ & 0.06102 & cubic inch $\left(\right.$ in $\left.^{3}\right)$ \\
\hline
\end{tabular}




\title{
An Interface for Simulating Radiative Transfer In and Around Volcanic Plumes with the Monte Carlo Radiative- Transfer Model McArtim
}

\author{
By Christoph Kern
}

\begin{abstract}
This report describes two software tools that, when used as front ends for the three-dimensional backward Monte Carlo atmospheric-radiative-transfer model (RTM) McArtim, facilitate the generation of lookup tables of volcanic-plume optical-transmittance characteristics in the ultraviolet/visiblespectral region. In particular, the differential optical depth and derivatives thereof (that is, weighting functions), with regard to a change in $\mathrm{SO}_{2}$ column density or aerosol optical thickness, can be simulated for a specific measurement geometry and a representative range of plume conditions. These tables are required for the retrieval of $\mathrm{SO}_{2}$ column density in volcanic plumes, using the simulated radiativetransfer/differential optical-absorption spectroscopic (SRT-DOAS) approach outlined by Kern and others (2012). This report, together with the software tools published online, is intended to make this sophisticated SRT-DOAS technique available to volcanologists and gas geochemists in an operational environment, without the need for an indepth treatment of the underlying principles or the low-level interface of the RTM McArtim.
\end{abstract}

\section{Introduction}

In recent years, the use of ultraviolet (UV) remote-sensing techniques for the detection and quantification of $\mathrm{SO}_{2}$ in volcanic plumes has become increasingly widespread (see review by Kantzas and Mcgonigle, 2008). These techniques rely on solar radiation scattered in the atmosphere as a light source for gas measurements. Conventionally, the solar radiation is assumed to propagate along a straight line from the scattering point, through the plume, to the instrument. This conceptual model, however, has been shown to fail when used to measure plumes rich in $\mathrm{SO}_{2}$ or with heavy aerosol loads, or when measuring $\mathrm{SO}_{2}$ distribution from a distance of more than 1 to 2 kilometers $(\mathrm{km})($ Kern and others, 2009). In such situations, a more complex model must be used to take into account the radiative transfer of the scene. Kern and others (2012) recently developed a retrieval technique called simulated radiative-transfer/differential optical-absorption spectroscopy (SRT-DOAS), which integrates moderateresolution spectroscopic measurements and radiances simulated by using the three-dimensional backward Monte Carlo atmospheric-radiative-transfer model (RTM) McArtim (Deutschmann and others, 2011). In this method, simulated radiances and weighting functions take the place of the absorption cross sections used in conventional DOAS retrievals (see Platt and Stutz, 2008, and references therein). Application of SRT-DOAS can substantially improve the accuracy of $\mathrm{SO}_{2}$ columndensity and emission-rate measurements (Kern and others, 2012). Thus, efficient generation of the requisite lookup tables permits rapid application of SRT-DOAS in an operational environment. 
In this report, a front end to the RTM McArtim is described that can be used to generate lookup tables containing these radiances and weighting functions for use in operational SRT-DOAS retrievals. In particular, two software programs are described: LT-Builder (Lookup Table Builder) and RTVP (Radiative Transfer in Volcanic Plumes). Both programs are interfaces for the RTM McArtim (version 2; Deutschmann and others, 2011), and both are freely available from the VHub Web site (https://vhub.org/). The published repositories each contain an appropriate release of the RTM McArtim.

The LT-Builder software is high-level code, written in the MATLAB programming language, that facilitates the simulation of spectral radiances, optical densities, and radiance derivatives in the UV spectral region (typically 300-340 nanometers [nm]) that are evaluated when applying UV remotesensing instrumentation to volcanic plumes. The name originates in the program's ability to batch process different volcanic-plume conditions, thus lending itself to the generation of lookup tables for use in SRT-DOAS retrievals, as described by Kern and others (2012).

The RTVP software is a low-level front end for the RTM McArtim that allows a parameterization of volcanic plumes for model initialization. RTVP is compiled $\mathrm{C}++$ code that can be called from the Windows command-line interpreter. RTVP provides more flexibility than is available through LT-Builder, yet it is considerably less user friendly when run as a stand-alone program. LTBuilder uses RTVP as an interface to the RTM McArtim, as shown schematically in figure 1. Users interested in simulating radiances, optical densities, and weighting functions - for example, for use in SRT-DOAS retrievals - will likely find LT-Builder to have sufficient functionality to meet their needs and may not need to familiarize themselves with RTVP. However, documentation for using RTVP as a stand-alone program is also provided for those users who require a more flexible, albeit less intuitive, front end to the RTM McArtim.

After simulations are run, the model output files $\left({ }^{*}\right.$.rtm and *.adr) are automatically translated to text files (*.txt) that contain spectral data. The various resulting spectrum types are described in the next section, but all the text files can easily be imported into any plotting software or, if further processing is required, into the DOAS Intelligent System (DOASIS) software. Some basic postprocessing steps in DOASIS are also described in this report.

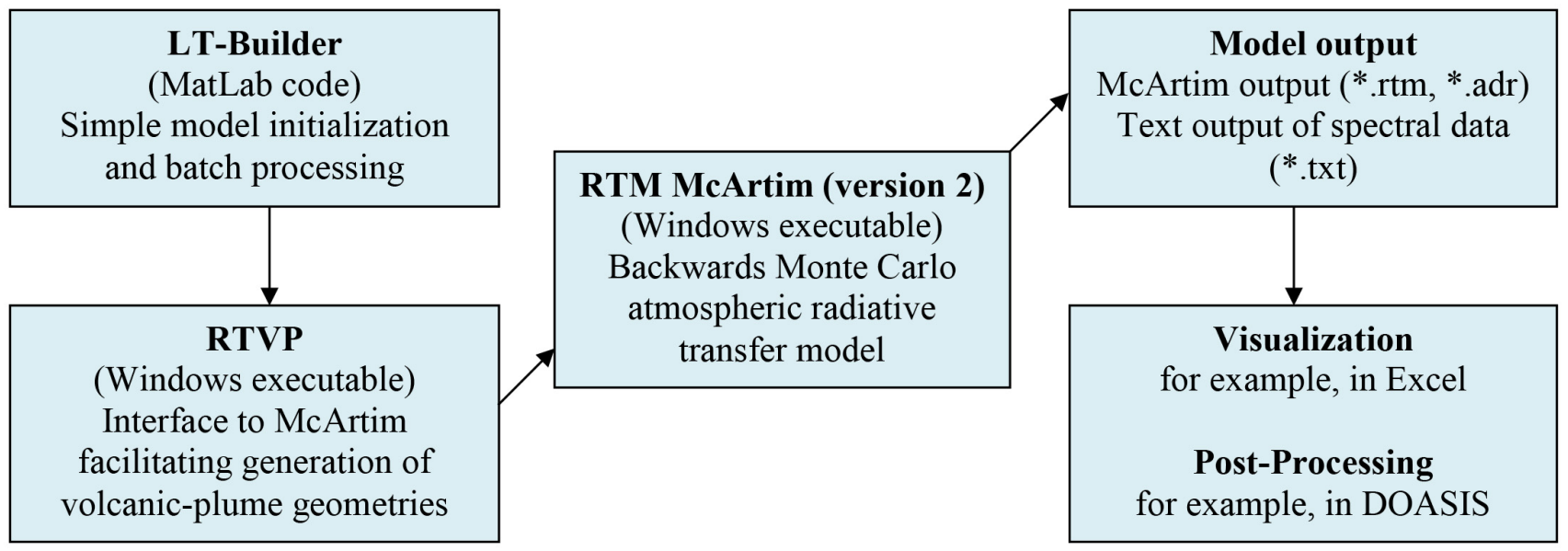

Figure 1. Diagram showing flow of information for simulating radiative transfer in and around volcanic plumes using the Lookup Table Builder (LT-Builder) and Radiative Transfer in Volcanic Plumes (RTVP) interfaces for the radiative transfer model (RTM) McArtim. 


\section{LT-Builder}

The LT-Builder software was designed as a simple tool for simulating spectral radiances, optical densities, and radiance derivatives in volcanic-plume geometries for use in sophisticated spectroscopic retrievals, particularly for SRT-DOAS approaches (Kern and others, 2012). In these retrievals, simulated optical densities and derivatives with regard to $\mathrm{SO}_{2}$ absorption and plume aerosol optical thickness (AOT) take the place of the absorption cross sections used in conventional DOAS retrievals. These more sophisticated retrievals are performed to improve the accuracy of derived column densities whenever three-dimensional radiative-transfer effects are significant (see Kern and others, 2009). To facilitate the fast evaluation of operational datasets, these retrievals require a library (lookup table) of optical densities and weighting functions simulated over a range of plume conditions. Although simulation of an entire lookup table is still time and computationally intensive, initializing the RTM McArtim for the generation of such a table is simple using LT-Builder. Here, each step of the process is explained, beginning with system requirements and installation. Examples are given, and postprocessing with the DOASIS software is explained.

\section{System Requirements}

- A 32-bit Microsoft Windows system, preferably on a multicore CPU platform

- LT-Builder software

The MATLAB LT-Builder software is used to iterate through different atmospheric conditions and thus build the lookup table. The program is kept as simple as possible and makes only the most basic RTM settings accessible.

- MathWorks MATLAB program

The LT-Builder software is written in the MATLAB programming language and is currently unavailable in a compiled version; therefore, the software needs the MATLAB interpreter to run. MATLAB can be purchased online from MathWorks (http://www.mathworks.com/products/matlab/); version 2008a or higher is required.

- RTVP software

The RTVP software is a front end, written in Microsoft Visual $\mathrm{C}++$, for the monochromatic RTM McArtim (version 2) that allows the simulation of spectra. RTVP was developed by the author and is included in the LT-Builder distribution package. Documentation for using RTVP as a stand-alone tool is presented in the next section.

- Microsoft Visual $\mathrm{C}++2010$ redistributable package

The Microsoft Visual $\mathrm{C}++2010$ redistributable package is required to run programs compiled with Microsoft Visual C++ 2010, in which the RTVP software was written. The package can be downloaded from Microsoft's Web site (http://www.microsoft.com/enus/download/details.aspx?id=5555). The package is not required if Microsoft Visual $\mathrm{C}++$ is already installed on the computer.

- McArtim (version 2)

McArtim (Deutschmann and others, 2011) is a backward Monte Carlo atmospheric radiative inversion transfer model developed at the University of Heidelberg, Germany. A compiled version of McArtim (version 2, compiled for Windows 32-bit systems) is included in the LTBuilder distribution package.

- DOASIS

DOASIS is a spectral acquisition and evaluation software developed at the University of Heidelberg, Germany. It is free of charge but requires registration. It is available for download 
from the DOASIS Web site (http://www.iup.uni-

heidelberg.de/institut/forschung/groups/atmosphere/software). DOASIS is not required for simulation of optical densities but is needed for building scattering weighting functions or convolving the simulated spectra with instrument line shapes of respective instruments. Several example DOASIS scripts are included in the LT-Builder distribution package.

\section{Installation and Directory Structure}

The LT-Builder software is written in the MathWorks MATLAB programming language and does not require installation on the system. It does rely on the availability of both RTVP and McArtim, which are included in the LT-Builder distribution package. To get started, unpack the zip file to the hard drive, which will automatically generate a base directory named LT-Builder (hereafter, the exact names of folders, files, classes, class methods and class parameters will be shown in italics. Also note the following slight differences in spelling. LT-Builder refers to the name of the software, LT-Builder refers to the name of the base folder, and LTBuilder refers to the name of a specific class. Similarly, RTVPSettings refers to the name of a specific class, while RtvpSettings refers to a handle pointing to that class.. This directory contains the MATLAB code and several subfolders. The LT-Builder code is very basic, consisting of only three files. One file, LTBuilder.m, defines the LTBuilder class. Once initialized, the class properties are then used to store the information required to build the lookup table, and the class methods can be used for setting up a directory structure and running the RTM. Another file, RTVPSettings.m, defines a second class that is used by the LTBuilder class to manipulate the settings of the RTVP program. It is automatically initialized by the LTBuilder class, so it can be considered a subclass.

The RTM McArtim (version 2, compiled for Windows) is included in the LT-Builder distribution package and is located in a subfolder named McArtim. The subfolder named RTVP will already contain the compiled RTVP program, which also is included in the LT-Builder distribution package. The subfolder named DOASIS scripts contains some example scripts that demonstrate the postprocessing of simulation results, as briefly described below. The subfolder named Output is the standard folder for output of the lookup tables, but this location can be changed by the user.

Because the LTBuilder class calls the RTVP front end, which was written in Microsoft Visual $\mathrm{C}++$, the Microsoft Visual $\mathrm{C}++$ redistribution package must be installed on the system before running LT-Builder. Also, if postprocessing of simulated spectra is needed, the DOASIS software may prove valuable. See the System Requirements section of this report for information on how to obtain these additional packages, both of which are distributed with dedicated Windows installers.

\section{Quick Reference-How to Simulate a Spectrum or Build a Lookup Table}

To simulate a spectrum or build a lookup table with the LTBuilder class, first define an instance of the class in MATLAB. For example, enter the following line into the MATLAB command interpreter:

$$
\text { LTBuilder }=\text { LTBuilder(); }
$$

Next, adapt the required settings to reflect the desired plume geometry and measurement setup. Finally, start the simulations by calling the method LTBuilder.RunRaytracer(). This procedure is demonstrated in the script example BuildLookupTableExample.m, which is included in the LT-Builder distribution package (fig. 2). 


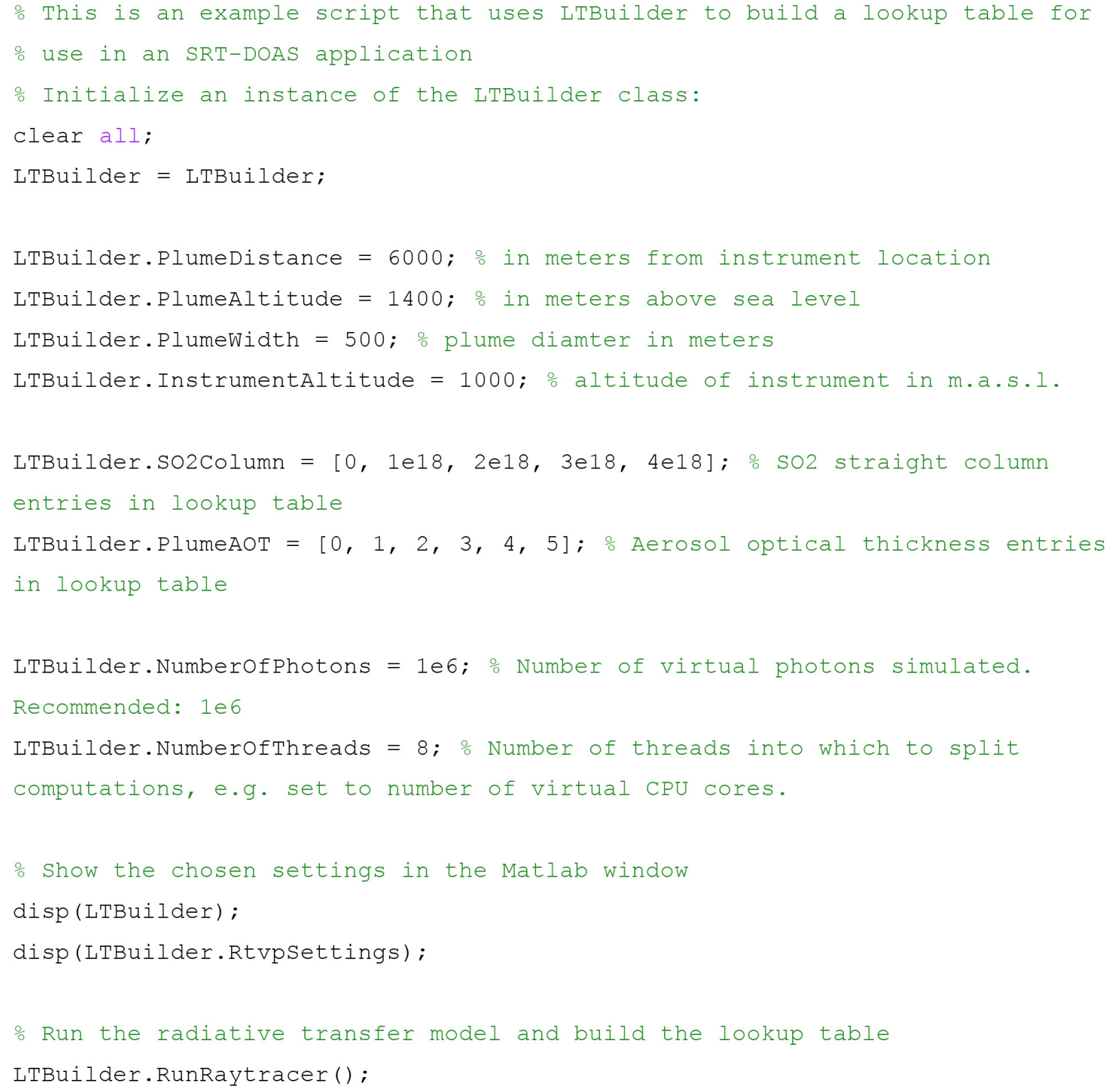

Figure 2. Example MatLab script that can be used to generate a lookup table for use in sophisticated spectroscopic retrievals. This script (BuildLookupTableExample.m) is included in the software distribution of the Lookup Table Builder (LT-Builder) software.

Running the example code in MatLab will generate a lookup table, simulating the spectral radiance, optical density, and absorption-weighting function for a specific geometry and range of plume $\mathrm{SO}_{2}$ column densities and AOTs (defined at $300 \mathrm{~nm}$ ). Note that the measurement and plume geometries are set to constant values, whereas the parameters named SO2Column and PlumeAOT are set to arrays of values in this example. These arrays define the individual positions of the lookup table; all combinations of $\mathrm{SO}_{2}$ straight-column density and plume AOT will be simulated. If a single spectrum is 
desired, rather than an entire table, the column density and AOT can be set to a single value instead of an array.

Other than the geometry and plume settings, few parameters are needed. The NumberOfPhotons parameter will determine the precision of the simulations. A low number $\left(\sim 10^{3}\right)$ can be used for quick testing, but a value of $10^{6}$ is recommended to obtain sufficient precision when building tables for use in SRT-DOAS retrievals. Note, however, that on typical desktop computers, simulation of a single lookuptable position with $10^{6}$ photons may take several hours. The processing speed for an entire table can be improved by assigning the different table positions to separate CPU cores. In the given example, the NumerOfThreads parameter was set to 8 , thus starting eight independent processes to take advantage of the eight virtual cores on this particular system.

Some additional settings are accessible as properties of the LTBuilder and RTVPSettings classes. In the given example, the default values were kept, but all the properties are displayed in the MATLAB window using the commands disp(LTBuilder) and disp(LTBuilder.RtvpSettings). Note that in this syntax, RtvpSettings is a handle to an instance of the RTVPSettings class. Therefore, the capitalization is different. Finally, the command RunRaytracer() is called to start the simulation. This method will create a subfolder named Threads in the LT-Builder base directory and copy various instances of the RTM McArtim and the RTVP program into it. Each instance will be initialized to process certain lookup-table positions, and then each instance will be started. A command-prompt window will appear for each instance in which the current model output is shown.

After all the model instances are started, MATLAB can be closed because it is no longer required to run the simulations. As they are obtained, the lookup-table entries will appear in the specified output directory. By default, this is the subfolder Output in the LT-Builder base directory. The filenames of the lookup-table entries begin with the specified geometry, according to the following naming convention: $<$ PlumeDistance $><$ PlumeAltitude $><$ PlumeWidth $><$ SO2Column $>$ $<$ PlumeAOT $><$ InstrumentAltitude $>$. *

The filename extension specifies the file type for the respective geometry. Some file types are internal RTM files, but the following file types are of special interest:

$<$ OutputFile>.meas.txt

$<$ OutputFile>.ref.txt

$<$ OutputFile>.od.txt

$<$ OutputFile>.adr.txt
The optical radiance simulated for a geometry looking toward the center of the plume.

The optical radiance simulated for a geometry looking away from or without a plume.

The optical depth obtained by dividing the radiance simulated outside the plume by the radiance simulated for the plume center and then applying the natural logarithm.

The weighting function with respect to $\mathrm{SO}_{2}$ absorption.

\section{Visualization and Postprocessing of Lookup-Table Entries}

The text files created by the LT-Builder program can be imported into any data-plotting program. The files contain two (tab-delimited) columns of ASCII data, the first of which is the wavelength, and the second of which represents the respective spectrum value. Of particular value is the ability to open the spectra directly with the DOASIS software, which can then be used to view the output, as well as to perform additional processing steps on the simulated spectra.

If, for example, the output is to be used for an SRT-DOAS evaluation, the weighting functions with regard to scattering in the plume will be required. These weighting functions can be derived from the difference quotients of the lookup-table entries once they have all been simulated. DOASIS provides a scripting functionality that allows quick building of these scattering derivatives for all lookup-table 
entries. LT_Build_SDR.js, which is a j-script file that performs this task, is included in the LT-Builder distribution package inside the subfolder named DOASIS scripts. A second j-script file,

$L T$ Convolute.js, provides an example of how the lookup-table entries can be convolved with the instrument line shape of a given spectrometer and mapped onto its wavelength grid. This operation is also required when using the model output to evaluate actual measurement data.

\section{Programming References}

The LT-Builder code is simple and most easily understood by using the quick reference provided in the previous section. However, for the sake of completeness, all properties and methods of the LTBuilder and the RTVPSettings classes are described in this section. Users unfamiliar with objectoriented programming in MATLAB may find the online MathWorks documentation on this topic useful (http://www.mathworks.com/discovery/object-oriented-programming.html). Additional help can be obtained by pressing F1 while the cursor is on a class, method, or property name in MATLAB.

\section{LTBuilder Class}

The LTBuilder class contains properties that store the most important information needed to simulate a lookup table, as well as the high-level methods needed to do so. Create an instance of this class, set the properties to the appropriate values, and start the simulation. Properties and methods available to the user are listed in tables 1 and 2.

Table 1. LTBuilder class public properties.

[LT-Builder, Lookup Table Builder; RTVP, Radiative Transfer in Volcanic Plumes; molecules/cm², molecules per square centimeter]

\begin{tabular}{|c|c|c|c|}
\hline Property & Type & Default value & Description \\
\hline BaseDir & string & $\mathrm{C}:\lfloor$ LT-Builder $\backslash$ & Base directory containing the LT-Builder software. \\
\hline RTMDir & string & $\mathrm{C}: \backslash \mathrm{LT}$-Builder $\backslash$ McArtim $\backslash$ & $\begin{array}{l}\text { Folder containing the radiative transfer model } \\
\text { McArtim. }\end{array}$ \\
\hline RTVPDir & string & C: $\backslash$ LT-Builder $\backslash$ RTVP $\backslash$ & Folder containing RTVP.exe. \\
\hline OutputDir & string & $\mathrm{C}: \backslash$ LT-Builder $\backslash$ Output $\backslash$ & Output folder for the lookup table. \\
\hline SO2Column & $\begin{array}{l}\text { array of } \\
\text { floats }\end{array}$ & {$[0,1 \mathrm{e} 18,2 \mathrm{e} 18,3 \mathrm{e} 18,4 \mathrm{e} 18]$} & $\begin{array}{l}\text { Array of } \mathrm{SO}_{2} \text { column densities to use in lookup- } \\
\text { table creation, in molecules } / \mathrm{cm}^{2} \text {. }\end{array}$ \\
\hline PlumeAOT & $\begin{array}{l}\text { array of } \\
\text { floats }\end{array}$ & {$[0,1,2,3,4,5]$} & $\begin{array}{l}\text { Array of plume aerosol optical depths to use in } \\
\text { lookup-table creation. }\end{array}$ \\
\hline PlumeDistance & int & 2,000 & Distance between instrument and plume (in meters). \\
\hline PlumeAltitude & int & 1,000 & Altitude of plume (in meters above sea level). \\
\hline PlumeWidth & int & 500 & Width of plume (in meters). \\
\hline InstrumentAltitude & int & 0 & Altitude of instrument (in meters above sea level). \\
\hline NumberOfThreads & int & 8 & $\begin{array}{l}\text { Number of threads into which to split the } \\
\text { computation-for example, set to number of } \\
\text { virtual CPU cores. }\end{array}$ \\
\hline RtvpSettings & $\begin{array}{l}\text { RTVPSetti } \\
\quad n g s\end{array}$ & See table 3 & $\begin{array}{l}\text { RTVPSettings class containing settings related to } \\
\text { the RTVP program. }\end{array}$ \\
\hline NumberOfPhotons & int & $10^{6}$ & Number of virtual photons to simulate. \\
\hline
\end{tabular}


Table 2. LTBuilder class public methods.

[LT-Builder, Lookup Table Builder; RTVP, Radiative Transfer in Volcanic Plumes; AOT, aerosol optical thickness]

Method

Description

\begin{tabular}{|c|c|}
\hline LTBuilder() & $\begin{array}{l}\text { Constructor of the LTBuilder class; also initializes several settings to default values; } \\
\text { returns LTBuilder. }\end{array}$ \\
\hline ThreadRollout() & $\begin{array}{l}\text { Creates a folder named Threads in the LTBuilder base directory. Here, it creates two } \\
\text { subfolders per thread - an instance of RTVP and an instance of the radiative-transfer } \\
\text { model. }\end{array}$ \\
\hline ThreadCleanup() & Removes the Threads folder with all of its subfolders. \\
\hline RunRaytracer() & $\begin{array}{l}\text { Cleans up the Threads folder, rolls out new instances of RTVP and the radiative-transfer } \\
\text { model, generates batch files for batch processing of the entries in the lookup table, and } \\
\text { then starts each batch file, each of which start an instance of the radiative-transfer } \\
\text { model. Command-prompt windows appear, one for each process. Once all processes } \\
\text { have been successfully started, MATLAB can be closed. }\end{array}$ \\
\hline AddLTEntries() & $\begin{array}{l}\text { Creates two text files in the output folder-one containing the } \mathrm{SO}_{2} \text { column densities that } \\
\text { are simulated in the lookup table and one containing the plume AOTs. These files are } \\
\text { later used to identify which entries exist in the lookup table. }\end{array}$ \\
\hline BuildBatchFiles() & $\begin{array}{l}\text { Generates a batch file for each instance of the RTVP program that successively calls the } \\
\text { required commands to generate the lookup-table entries. }\end{array}$ \\
\hline CheckBkSl(string) & $\begin{array}{l}\text { Checks whether the final character in the input text string is a backslash; if not, it adds } \\
\text { one. The method returns the string with a backslash at the end. }\end{array}$ \\
\hline
\end{tabular}

\section{RTVPSettings Class}

The RTVPSettings class is used to store settings that are written to the initialization file of the RTVP program. An instance of RTVPSettings is created on construction of the LTBuilder class. In most cases, these settings can be left unchanged. If changes are desired, be sure to call the WriteSettings() method to save them to disk. (Note that this method is also called operationally by LTBuilder.ThreadRollout().) Changes to RTVP_settings.ini will be saved and used as defaults in future instances of LTBuilder. The interface of the RTVPSettings class is described in tables 3 and 4.

Table 3. RTVPSettings class public properties.

[RTVP, Radiative Transfer in Volcanic Plumes; AOT, aerosol optical thickness; LT-Builder, Lookup Table Builder]

\begin{tabular}{|c|c|c|c|}
\hline Property & Type & Default value & Description \\
\hline RTVPPath & string & C: $\backslash$ LT-Builder $\backslash R T V P \backslash$ & $\begin{array}{l}\text { Folder containing the RTVP program } \\
\text { and settings files. }\end{array}$ \\
\hline NumberOfPhotons & int & $1 \mathrm{e} 6$ & $\begin{array}{l}\text { Number of virtual trajectories to } \\
\text { simulate. }\end{array}$ \\
\hline UseGaussianPlumeCrossSection & bool & 0 & $\begin{array}{l}0, \text { homogeneous } \mathrm{SO}_{2} \text { and aerosol } \\
\text { concentrations in the plume; } \\
1 \text {, Gaussian distribution. }\end{array}$ \\
\hline AngstromExponent & float & 1.2 & $\begin{array}{l}\text { Angstrom exponent for plume aerosol } \\
\text { scattering. }\end{array}$ \\
\hline SpectralResolution & float & 0.05 & $\begin{array}{l}\text { Spectral resolution (in nanometers) at } \\
\text { which to simulate the lookup table. }\end{array}$ \\
\hline
\end{tabular}




\begin{tabular}{|c|c|c|c|}
\hline Property & Type & Default value & Description \\
\hline SpectrumStartWavelength & float & 300 & $\begin{array}{l}\text { Lower end of the wavelength window } \\
\text { (in nanometers) in which to simulate } \\
\text { spectral radiances. }\end{array}$ \\
\hline SpectrumEndWavelength & float & 340 & $\begin{array}{l}\text { Upper end of the wavelength window (in } \\
\text { nanometers) in which to simulate } \\
\text { spectral radiances. }\end{array}$ \\
\hline ExternalReferenceSpectrum & string & $\begin{array}{l}\text { C: }: \text { LT-Builder } \backslash \text { RTVP } \backslash \\
\text { References } \backslash \text { null_ref_300- } \\
\text { 340nm-0.05nm.txt }\end{array}$ & $\begin{array}{l}\text { Filename of a fixed external reference } \\
\text { that contains the spectral radiance in } \\
\text { the absence of } \mathrm{SO}_{2} \text { or aerosols. Note } \\
\text { that the number of spectral channels } \\
\text { must match the wavelength range of } \\
\text { the simulation. Additional references } \\
\text { can be simulated by specifying } 0 \mathrm{SO}_{2} \\
\text { and AOT in } L T B u i l d e r \text {, and selecting a } \\
\text { new wavelength range. }\end{array}$ \\
\hline RTMPrimaryBaseDir & string & C:\LT-Builder $\backslash M c A r t i m \backslash$ & $\begin{array}{l}\text { Folder containing the radiative-transfer } \\
\text { model McArtim. }\end{array}$ \\
\hline OutputDir & string & C:LLT-Builder \Output $\backslash$ & Output folder for RTVP results. \\
\hline
\end{tabular}

Table 4. RTVPSettings class public methods.

[RTVP, Radiative Transfer in Volcanic Plumes]

\begin{tabular}{ll}
\hline \multicolumn{1}{c}{ Method } & Description \\
\hline RTVPSettings( & Constructor of the RTVPSettings class; returns an instance of the class. \\
ReadSettings() & Reads selected settings from the file $R T V P$ settings.ini in the folder specified by the \\
RTVPPath property; settings are loaded into the properties described in the previous \\
table. \\
Writes the settings stored in the RTVPSettings class properties to the file \\
RTVP_settings.ini located in the folder specified by the RTVPPath property.
\end{tabular}

Note that the RTVPSettings class does not access all the settings and functionality that the RTVP low-level front end for the RTM McArtim provides; therefore, it is not designed as a complete interface for use of RTVP as a standalone program. Instead, the RTVPSettings class acts as an interface between the LTBuilder class and the RTVP program, making the settings needed by the LTBuilder class accessible.

The low-level front end RTVP for the RTM McArtim can, however, be used as a stand-alone program by calling it from the command line. Although this method should not be needed for users simply seeking to calculate spectral radiances and(or) lookup tables for use with SRT-DOAS, it may be suitable to users looking for more flexibility in their application of McArtim to volcanic-plume scenarios. Therefore, documentation of RTVP is provided in the following section.

\section{RTVP}

RTVP is a low-level front end for the RTM McArtim. Written and compiled in Microsoft Visual $\mathrm{C}++$, RTVP provides a simple command-line interface for users to initialize and run McArtim in geometries relevant for the volcano-emissions remote-sensing community. However, users interested 
only in a simple means of simulating spectral radiances, optical densities, and weighting functions, for example for use in SRT-DOAS retrievals (as described by Kern and others, 2012) may not need to familiarize themselves with RTVP. Instead, a high-level front end specifically written for this application is available in the form of a MATLAB program (see previous section).

\section{System Requirements}

- Microsoft Windows operating system

- Microsoft Visual C++ Redistributable Package installed (available from http:/www.microsoft.com/en-us/download/details.aspx?id=5555)

- Compiled RTVP package

- McArtim RTM, version 2, compiled for 32-bit Windows operating system (included in the RTVP distribution package)

\section{Installation and Directory Structure}

Because RTVP is compiled to an executable file, it does not require installation. To begin, unpack the zip file to a directory on the hard drive; the base directory will contain the file RTVP.exe. This executable file can be called from the Windows Command Prompt; the syntax for doing so is described in the next section. The file RTVP_settings.ini, also contained in the base directory, holds settings for the RTM, which are also discussed below. A folder named McArtim will be generated in the base directory; this folder contains two subfolders (Raytracel and Raytrace2), each of which contains an instance of McArtim. Depending on how the RTVP program is used, one or both of these instances may be called simultaneously. The Results folder can be used as a target for RTM output, and the Scenarios folder contains an example measurement scenario, as shown in the examples described below.

\section{Running RTVP from the Command Prompt}

As mentioned above, RTVP is compiled to run from the Windows Command Prompt. Launch a new command prompt by clicking 'Start' and typing "cmd". As the RTVP folder is most likely not in the system path, navigate to the folder in which the file RTVP.exe is located by using the commands $c d$.. (back up one directory level) and $c d$ [dir] (change directory). Once the RTVP base directory is active, typing rtvp followed by Enter will return syntax information regarding the use of RTVP as a front end for McArtim. The general syntax is

\section{rtvp-[operation mode] [command line variables]}

The operation mode defines the behavior of McArtim; individual operation modes are described in the next section. The command-line variables are parameters that define the geometry of the simulation and tell McArtim where to write the model output files. As described later, the required command-line variables change depending on the operation mode.

\section{RTVP Operation Modes}

The first parameter following rtvp on the command line represents the RTVP operation mode, a three- or four-letter identifier, preceded by a hyphen, that defines what the output of the RTM should be. The valid operation modes, along with the command-line variables required for each operation mode, are listed in table 5. The individual command-line variables are discussed in the next section. 
Table 5. RTVP operation modes.

[RTVP, Radiative Transfer in Volcanic Plumes; DOAS, differential optical-absorption spectroscopy; AOT, aerosol optical thickness]

\begin{tabular}{cc}
\hline $\begin{array}{c}\text { Mode } \\
\text { identifier }\end{array}$ Command-line variables & Description of operation mode \\
\hline
\end{tabular}

$\begin{array}{ll} & \text { Wavelength(nm) } \\ & \text { PlumeDistance }(\mathrm{m}) \\ & \text { PlumeAltitude(m.a.s.1.) } \\ \text {-scd } & \text { PlumeDiameter(m) } \\ & \mathrm{SO}_{2} \text { Column}\left(\operatorname{molec} / \mathrm{cm}^{2}\right) \\ & \text { PlumeAOT(300nm) } \\ & \text { InstrumentAltitude(m.a.s.1.) } \\ & \text { OutputFile }\end{array}$

PlumeDistance $(\mathrm{m})$

PlumeAltitude(m.a.s.1.)

PlumeDiameter(m)

$\mathrm{SO}_{2}$ Column $\left(\right.$ molec $\left./ \mathrm{cm}^{2}\right)$

-odd PlumeAOT(300nm)

InstrumentAltitude(m.a.s.1.)

ReferenceElevationAngle(deg)

LineShapeFile

OutputFile

$\begin{array}{ll} & \text { PlumeDistance(m) } \\ & \text { PlumeAltitude(m.a.s.l.) } \\ & \text { PlumeDiameter(m) } \\ & \left.\text { SO }{ }_{2} \text { Column(molec/cm }{ }^{2}\right) \\ \text {-ods } & \text { PlumeAOT(300nm) } \\ & \text { InstrumentAltitude(m.a.s.1.) } \\ & \text { ReferenceElevationAngle(deg) } \\ & \text { LineShapeFile } \\ & \text { WavelengthSelectionFine } \\ & \text { OutputFile } \\ & \\ & \text { PlumeDistance(m) } \\ & \text { PlumeAltitude(m.a.s.l.) } \\ & \text { PlumeDiameter(m) } \\ & \left.\text { InitialSO }{ }_{2} \text { Column(molec/cm }{ }^{2}\right) \\ & \text { InitialPlumeAOT(300nm) } \\ & \text { InstrumentAltitude(m.a.s.l.) } \\ & \text { ReferenceElevationAngle(deg) } \\ & \text { MeasurementSpectrum } \\ \text { ReferenceSpectrum } & \text { CalibrationFile } \\ \text { LineShapeFile } & \text { OutputFile } \\ & \end{array}$

PlumeDistance(m)

PlumeAltitude(m.a.s.1.)

PlumeDiameter $(\mathrm{m})$

$\mathrm{SO}_{2}$ Column $\left(\mathrm{molec} / \mathrm{cm}^{2}\right)$

InstrumentAltitude(m.a.s.1.)

ReferenceElevationAngle(deg)

WavelengthSelectionFine

OutputFile

PlumeDistance $(\mathrm{m})$

de(m.a.s.l.)

PlumeDiameter $(\mathrm{m})$

InitialPlumeAOT $(300 \mathrm{~nm})$

InstrumentAltitude(m.a.s.l.)

ReferenceSpectrum

LineShapeFile

OutputFile
Runs RTVP in slant column density mode. In this mode, RTVP simulates the slant column density of $\mathrm{SO}_{2}$ that an instrument would detect for a single given wavelength and a fixed measurement scenario. A DOAS instrument cannot derive a column density from a single wavelength but instead uses a range of wavelengths. The model can simulate the column for any wavelength.

Runs RTVP in optical density mode using discrete wavelengths. In this mode, RTVP simulates an optical density spectrum for a given measurement scenario. Both measurement and reference spectra are simulated at discrete, equally spaced wavelengths. The spectral window and simulated wavelength resolution are set in file RTVP_settings.ini.

Runs RTVP in optical density mode using specific wavelengths. In this mode, RTVP simulates an optical density spectrum for a given measurement scenario. Both the measurement and reference spectra are simulated for specific wavelengths given as a list in a text file. The filename of the wavelength file is passed as a command-line variable.

Runs RTVP in inversion mode. In this mode, RTVP varies the atmospheric conditions in an attempt to minimize the discrepancy between measured and modeled optical density spectra. In addition to the measurement conditions, measured plume and reference spectra are passed to the raytracer as command-line variables. Note: This operation mode is not yet validated and is provided only as a beta version. 


\begin{tabular}{cc}
\hline $\begin{array}{c}\text { Mode } \\
\text { identifier }\end{array}$ Command-line variables & Description of operation mode \\
\hline
\end{tabular}

PlumeDistance(m)

PlumeAltitude(m.a.s.l.)

PlumeDiameter(m)

InstrumentAltitude(m.a.s.1.)

ReferenceElevationAngle(deg)

MeasurementSpectrum

ReferenceSpectrum

-chi2 CalibrationFile

LineShapeFile

LowSO $\mathrm{S}_{2}$ Column $\left(\mathrm{molec} / \mathrm{cm}^{2}\right)$

$\mathrm{HighSO}_{2}$ Column $\left(\mathrm{molec} / \mathrm{cm}^{2}\right)$

$\mathrm{SO}_{2}$ ColumnResolution $\left(\mathrm{molec} / \mathrm{cm}^{2}\right)$

LowPlumeAOT

HighPlumeAOT

PlumeAOTResolution

OutputFile
Generates a topography of $\mathrm{CHI}^{2}$ values depicting the difference between a single specific measured optical depth and the optical depth simulated for various plume conditions (see Kern and others, 2012). The measured optical depth is given by passing a measurement and reference spectrum, and the limits of the topography are set by passing the range and resolution of $\mathrm{SO}_{2}$ and AOT values to consider. Note: This operation mode is not yet validated and is provided only as a beta version.

\section{RTVP Command-Line Variables}

RTVP has two kinds of settings; those that are passed to the program by using the command line and those that are configured in the initialization file RTVP_settings.ini. The settings passed by using the command line are generally those that define the plume geometry and $\mathrm{SO}_{2} /$ aerosol content, whereas those configured in the initialization file are typically not changed so often. Each program operation mode requires its own specific set of command-line variables; those that are required can be queried by typing rtvp at the command line or consulting table 5. Note that the correct number of variables in the correct order must always be provided for each operation mode. If the number of variables does not match the mode's requirements, the basic syntax information is displayed, and the model is not run. The various RTVP command-line variables are listed in table 6.

Table 6. RTVP command-line variables.

[AOT, aerosol optical thickness; nm, nanometers]

Variable

Description

PlumeDistance

Distance (in meters) between the instrument and the center of the volcanic plume.

PlumeAltitude

PlumeDiameter

InstrumentAltitude

ReferenceElevationAngle
Altitude (in meters above sea level) of the volcanic plume.

Diameter (in meters) of the volcanic plume (assumed to have a circular cross section).

Assumed altitude (in meters above sea level) of the remote-sensing instrument. The elevation angle of the instrument-viewing direction is chosen such that it points toward the center of the plume.

Elevation angle (in degrees above the horizon) used to simulate a reference spectrum for the measurement (unless a fixed reference is used — see settings in the initialization file). 


\begin{tabular}{|c|c|}
\hline Variable & Description \\
\hline MeasurementSpectrum & $\begin{array}{l}\text { Path and file name of a measured plume spectrum. Spectrum must be in the *.std ASCII } \\
\text { format (see examples below). }\end{array}$ \\
\hline ReferenceSpectrum & $\begin{array}{l}\text { Path and file name of a measured Fraunhofer reference spectrum. Spectrum must be in } \\
\text { *.std ASCII format (see examples below). }\end{array}$ \\
\hline CalibrationFile & $\begin{array}{l}\text { Path and file name of a calibration text file. File must contain a list of wavelengths } \\
\text { corresponding to each channel of the spectrometer. }\end{array}$ \\
\hline LineShapeFile & $\begin{array}{l}\text { Path and file name of the instrument line-shape file. File must contain a single column of } \\
\text { ASCII data describing the instrument response to a monochromatic light source, } \\
\text { calibrated according to the calibration file above. }\end{array}$ \\
\hline InitialSO ${ }_{2}$ Column & $\begin{array}{l}\text { Initial } \mathrm{SO}_{2} \text { column density (in molecules per square centimeter) considered during } \\
\text { inversion of this parameter in the inv operating mode. }\end{array}$ \\
\hline InitialPlumeAOT & $\begin{array}{l}\text { Initial plume AOT (determined at } 300 \mathrm{~nm} \text { ) considered during inversion of this parameter } \\
\text { in the inversion operating mode. }\end{array}$ \\
\hline LowSO2Column & $\begin{array}{l}\text { Lower limit of } \mathrm{SO}_{2} \text { column densities (in molecules per square centimeter) considered in } \\
\text { chi } 2 \text { mode. }\end{array}$ \\
\hline HighSO2Column & $\begin{array}{l}\text { Upper limit of } \mathrm{SO}_{2} \text { column densities (in molecules per square centimeter) considered in } \\
\text { chi2 mode. }\end{array}$ \\
\hline SO2ColumnResolution & $\begin{array}{l}\text { Resolution of } \mathrm{SO}_{2} \text { column densities (in molecules per square centimeter) considered in } \\
\text { chi2 mode. }\end{array}$ \\
\hline LowPlumeAOT & Lower limit of plume AOTs (determined at $300 \mathrm{~nm}$ ) considered in chi2 mode. \\
\hline HighPlumeAOT & Upper limit of plume AOTs (determined at $300 \mathrm{~nm}$ ) considered in chi2 mode. \\
\hline PlumeAOTResolution & Resolution of plume AOTs (determined at $300 \mathrm{~nm}$ ) considered in chi2 mode. \\
\hline WavelengthSelectionFile & File containing a list of wavelengths at which the simulation will be run. \\
\hline OutputFile & $\begin{array}{l}\text { Path and file name for results of the simulation. Several files will be created, with } \\
\text { variations in the file extension, indicating the type of result (see quick reference for } \\
\text { examples). }\end{array}$ \\
\hline
\end{tabular}

\section{Scenario Files}

Instead of passing all geometry variables to RTVP on the command line, scenario files can be created that contain the geometry settings. Scenario files contain all of the variables described above. An example scenario file, named example_scenario.txt, is included in the RTVP distribution package, in the Scenarios subfolder. Any text editor can be used to make changes to the scenario file, but the number and order of the entries in the scenario file must be maintained (even if not all entries are used in the specific operation mode), and the last entry must be followed by a carriage return (blank line).

After the scenario parameters are adjusted by editing the scenario file, RTVP is called by using the name of the scenario file in place of the command-line variables:

\section{rtvp-[operation mode] [scenario file]}




\section{Settings in the Initialization File}

Besides the settings passed to RTVP by way of the command line, additional settings are defined in the RTVP_settings.ini file. The various entries in $R T V P \_$settings.ini are listed in table 7 . Any text editor can be used to edit this file. Note that the order of entries may not be changed while editing, and the last entry must be followed by a carriage return (blank line). Most of these entries do not need to be changed because the default values are appropriate in most cases and some parameters were introduced for debugging purposes only. Parameters that may need to be adjusted by the user are as follows:

- RTM_PRIMARY_BASEDIR_contains the path to the first instance of McArtim and must be set by the user to match the folder in which McArtim is located on the current system.

- RTM_SECONDARY_BASEDIR_contains the path to the second instance of McArtim and must be set by the user to match the folder in which McArtim is located on the current system.

- RTM_OUTPUT_DIR_contains the path to the output folder into which results will be saved; this folder must exist on the current system.

- RTM_NUMBEROFPHOTONS — sets the number of virtual trajectories simulated by the McArtim; more trajectories lead to a more precise result but take longer to simulate.

- RTM_USE_GAUSSIAN_PLUME_CROSS_SECTION_toggles the $\mathrm{SO}_{2}$ concentration and $\mathrm{AOT}$ in the plume cross section between homogeneous (set to 0 ) or Gaussian (set to 1) distribution.

- RTM_ANGSTROM_EXPONENT_Angstrom exponent for aerosol scattering within the plume.

- RTVP_ODD_SPECTRAL_RESOLUTION_-Resolution used for spectra simulated in optical sensity mode using siscrete wavelengths (ODD mode, see description of program modes in table $5)$.

- RTVP_ODD_INV_BEGIN_SPECTRUM_SIMULATION_lower limit of wavelength range simulated in ODD mode.

- RTVP_ODD_INV_END_SPECTRUM_SIMULATION_ upper limit of wavelength range simulated in ODD mode.

Table 7. All settings configured in the RTVP_settings.ini initialization file (RTVP version 1.0.0). [AOT, aerosol optical thickness; ODD, Optical Density using Discrete wavelengths; INV, inversion]

\begin{tabular}{|c|c|c|c|c|}
\hline Parameter & Type & Default value & Mode & Description \\
\hline RTVP_SEND_OUTPUT_TO_FILE & bool & 0 & all & Toggle the logfile on/off. \\
\hline RTVP_OUTPUT_FILE & string & RTVP_out.txt & all & Logfile name. \\
\hline RTM_PRIMARY_BASEDIR & string & $\begin{aligned} \mathrm{C}: & \lfloor\mathrm{RTVP} \backslash \\
& \text { McArtim } \backslash \\
& \text { Raytrace1 }\end{aligned}$ & all & $\begin{array}{l}\text { Path to first instance of the RTM } \\
\text { McArtim. }\end{array}$ \\
\hline RTM_SECONDARY_BASEDIR & string & $\begin{aligned} & \mathrm{C}:\lfloor\mathrm{RTVP} \backslash \\
& \mathrm{McArtim} \backslash \\
& \text { Raytrace } 2\end{aligned}$ & all & $\begin{array}{l}\text { Path to second instance of the } \\
\text { RTM McArtim. }\end{array}$ \\
\hline RTM_OUTPUTDIR & string & $\begin{array}{r}\mathrm{C}: \mid \mathrm{RTVP} \backslash \\
\text { Results } \backslash\end{array}$ & all & Output path for results. \\
\hline
\end{tabular}




\begin{tabular}{|c|c|c|c|c|}
\hline Parameter & Type & Default value & Mode & Description \\
\hline RTM_NUMBEROFPHOTONS & int & $1 \mathrm{e} 6$ & all & $\begin{array}{l}\text { Number of simulated } \\
\text { trajectories. }\end{array}$ \\
\hline RTM_SAVE_TRAJECTORIES & bool & 0 & all & Toggle save trajectories to disk. \\
\hline $\begin{array}{l}\text { RTM_NUMBER_OF_VOXALS_IN_PL } \\
\text { UME_DIAMETER }\end{array}$ & int & 21 & all & Spatial resolution of the plume. \\
\hline $\begin{array}{l}\text { RTM_USE_GAUSSIAN_PLUME_CRO } \\
\text { SS_SECTION }\end{array}$ & bool & 0 & all & $\begin{array}{l}\text { Toggle Gaussian distribution of } \\
\mathrm{SO}_{2} \text { and aerosols in the plume. } \\
\text { Default is homogeneous } \\
\text { distribution. }\end{array}$ \\
\hline RTM_ANGSTROM_EXPONENT & float & 1.2 & all & $\begin{array}{l}\text { Angstrom exponent of aerosol } \\
\text { scattering. }\end{array}$ \\
\hline $\begin{array}{l}\text { RTVP_ODD_SPECTRAL_RESOLUTI } \\
\text { ON }\end{array}$ & float & 0.05 & odd & $\begin{array}{l}\text { Spectral resolution of simulation } \\
\text { in ODD mode. }\end{array}$ \\
\hline $\begin{array}{l}\text { RTVP_ODD_INV_BEGIN_SPECTRU } \\
\text { M_SIMULATION }\end{array}$ & float & 300 & odd, inv & $\begin{array}{l}\text { Lower limit of wavelength range } \\
\text { (in nanometers) during } \\
\text { spectrum simulation in ODD } \\
\text { mode. }\end{array}$ \\
\hline $\begin{array}{l}\text { RTVP_ODD_INV_END_SPECTRUM_ } \\
\text { SIMULATION }\end{array}$ & float & 340 & odd, inv & $\begin{array}{l}\text { Upper limit of wavelength range } \\
\text { (in nanometers) during } \\
\text { spectrum simulation in ODD } \\
\text { mode. }\end{array}$ \\
\hline $\begin{array}{l}\text { RTVP_OD_INV_CHI2_CONVOLUTE } \\
\text {-WITH_ILS }\end{array}$ & bool & 0 & $\begin{array}{l}\text { odd, ods, } \\
\text { chi2, } \\
\text { inv }\end{array}$ & $\begin{array}{l}\text { Toggle convolution with } \\
\text { instrument slit function on/off. }\end{array}$ \\
\hline $\begin{array}{l}\text { RTVP_OD_INV_CHI2_USE_FIXED_E } \\
\text { XTERNAL_REFERENCE_SPEC }\end{array}$ & bool & 0 & $\begin{array}{l}\text { odd, ods, } \\
\text { chi2, } \\
\text { inv }\end{array}$ & $\begin{array}{l}\text { Toggle use of an external } \\
\text { reference spectrum on/off. }\end{array}$ \\
\hline $\begin{array}{l}\text { RTVP_OD_INV_CHI2_EXTERNAL_R } \\
\text { EFERENCE_SPEC_FILE }\end{array}$ & string & $\begin{aligned} \mathrm{C}: & \backslash \mathrm{RTVP} \backslash \mathrm{Refs} \\
& \text { l300- } \\
& 340 \mathrm{~nm}- \\
& 0.05 \mathrm{~nm} . \mathrm{txt}\end{aligned}$ & $\begin{array}{l}\text { odd, ods, } \\
\text { chi2, } \\
\text { inv }\end{array}$ & $\begin{array}{l}\text { Path and file name of external } \\
\text { reference spectrum (number of } \\
\text { channels must match the } \\
\text { current simulation). }\end{array}$ \\
\hline $\begin{array}{l}\text { RTVP_INV_CHI2_STARTING_DAMP } \\
\text { ING_FACTOR }\end{array}$ & float & $1 \mathrm{e}-3$ & inv & $\begin{array}{l}\text { Damping factor }(\gamma) \text { in Levenberg } \\
\text { Marquardt inversion (INV } \\
\text { mode). }\end{array}$ \\
\hline $\begin{array}{l}\text { RTVP_INV_CHI2_DAMPING_FACTO } \\
\text { R_MULTIPLIER }\end{array}$ & float & 3 & inv & $\begin{array}{l}\text { Multiplier for damping factor in } \\
\text { Levenberg Marquardt } \\
\text { inversion. }\end{array}$ \\
\hline $\begin{array}{l}\text { RTVP_INV_AOD_DAMPING_ENHA } \\
\text { NCEMENT_FACTOR }\end{array}$ & float & 1 & inv & $\begin{array}{l}\text { Damping factor enhancement for } \\
\text { aerosol optical depth (as } \\
\text { opposed to } \gamma \text { for } \mathrm{SO}_{2} \text { column } \\
\text { density). }\end{array}$ \\
\hline $\begin{array}{l}\text { RTVP_INV_CHI2_USE_ONLY_SELE } \\
\text { CTED_WAVELENGTHS }\end{array}$ & bool & 0 & inv, chi2 & $\begin{array}{l}\text { Toggle selection of specific } \\
\text { wavelengths for spectrum } \\
\text { simulation (as opposed to } \\
\text { discrete wavelengths as in } \\
\text { ODD mode) on/off. }\end{array}$ \\
\hline
\end{tabular}




\begin{tabular}{|c|c|c|c|c|}
\hline Parameter & Type & Default value & Mode & Description \\
\hline $\begin{array}{l}\text { RTVP_INV_CHI2_WAVELENGTH_S } \\
\text { ELECTION_FILE }\end{array}$ & string & $\begin{array}{l}\mathrm{C}: \backslash \mathrm{RTVP} \backslash \mathrm{refs} \backslash \\
\text { SelectedWL } \\
\overline{\mathrm{SO}} \text { 2.txt }\end{array}$ & inv, chi2 & $\begin{array}{l}\text { Wavelength selection file (used } \\
\text { only if above parameter is set } \\
\text { to } 1 \text { ). }\end{array}$ \\
\hline $\begin{array}{l}\text { RTVP_INV_CHI2_STOP_CONDITION } \\
\text { _IF_CHI2_SMALLER_THAN }\end{array}$ & float & $1 e-6$ & inv, chi2 & $\begin{array}{l}\text { Stop condition for Levenberg- } \\
\text { Marquardt inversion of } \mathrm{SO}_{2} \\
\text { and AOT (INV mode). }\end{array}$ \\
\hline$\underset{\mathrm{S}}{\mathrm{RTVP}}$ & int & 20 & inv, chi2 & $\begin{array}{l}\text { Maximum iterations in } \\
\text { Levenberg } \mathrm{Marquardt} \\
\text { inversion of } \mathrm{SO}_{2} \text { and } \mathrm{AOT} \text {. }\end{array}$ \\
\hline $\begin{array}{l}\text { RTVP_INV_CHI2_FIT_POLYNOMIA } \\
\text { L_ORDER }\end{array}$ & int & 3 & inv, chi2 & $\begin{array}{l}\text { Order of additional polynomial } \\
\text { to fit to measurement data. }\end{array}$ \\
\hline $\begin{array}{l}\text { RTVP_INV_ALLOW_RETROACTIVE } \\
\text { ADJUSTMENT_OF_POLYNOMIA } \\
\overline{\mathrm{L}}\end{array}$ & bool & 1 & inv & $\begin{array}{l}\text { Additionally adjusts polynomial } \\
\text { for best after each iteration of } \\
\text { inversion. }\end{array}$ \\
\hline RTVP_INV_START_P0 & float & 0 & inv & $\begin{array}{l}\text { Initial value of fit polynomial } \\
\text { coefficient } 0 \text {. }\end{array}$ \\
\hline RTVP_INV_START_P1 & float & 0 & inv & $\begin{array}{l}\text { Initial value of fit polynomial } \\
\text { coefficient } 1 \text {. }\end{array}$ \\
\hline RTVP_INV_START_P2 & float & 0 & inv & $\begin{array}{l}\text { Initial value of fit polynomial } \\
\text { coefficient } 2 \text {. }\end{array}$ \\
\hline RTVP_INV_START_P3 & float & 0 & inv & $\begin{array}{l}\text { Initial value of fit polynomial } \\
\text { coefficient } 3 \text {. }\end{array}$ \\
\hline
\end{tabular}

\section{Examples}

In this section, a few examples are given to demonstrate how RTVP can be used as a front end to McArtim in order to simulate radiative transfer in and around a volcanic plume. Several operation modes are demonstrated. As discussed above, the behavior of the McArtim will differ in dependence on the RTVP operation mode. Note that simulation times will vary in dependence on system performance.

\section{Slant Column Density Program Mode}

An example call of RTVP in Slant Column Density (SCD) mode would be

$$
\text { rtvp -scd } 315300010007001 \text { 1e18 } 10 \text { testout.dat }
$$

Upon pressing Enter, RTVP will start the raytracer and simulate the column density of $\mathrm{SO}_{2}$ at $315 \mathrm{~nm}$ measured from a location $3 \mathrm{~km}$ from the plume center. In this scenario, the plume would be placed at $1 \mathrm{~km}$ above sea level, and its diameter would be $700 \mathrm{~m}$. The $\mathrm{SO}_{2}$ straight-column density, which is the same as the vertical-column density for a round-plume cross section like this, was chosen to be $1 \times 10^{18}$ molecules $/ \mathrm{cm}^{2}$. For a homogeneous plume, this value amounts to an $\mathrm{SO}_{2}$ concentration of $1.43 \times 10^{13}$ molecules $/ \mathrm{cm}^{3}$ in the plume. The plume aerosol optical depth is 1 , so a homogeneous plume would contain a scattering aerosol with an aerosol extinction coefficient of $1.43 \mathrm{~km}^{-1}$. Finally, the results will be written to the ASCII file testout.dat. 
Check the output file. In this particular example, the simulated $\mathrm{SO}_{2}$ column density should be $\sim 8.4 \times 10^{17}$ molecules $/ \mathrm{cm}^{2}, \sim 16$ percent lower than the true column, owing to radiative dilution occurring between the plume and the instrument. Because McArtim is a Monte Carlo model, the results could differ slightly when the model is run a second time. A higher precision can be obtained by running the model multiple times, or by increasing the number of simulated photons in the RTVP_settings.ini file.

\section{Optical Density at Discrete Wavelengths Program Mode}

For the next example, set the number of photons to 1,000 by editing the RTVP_settings.ini file. The ODD (Optical Density using Discrete wavelengths) program mode can be used to simulate an entire optical-density spectrum:

$$
\text { rtvp -odd } 300010007001 \text { le18 } 1090 \text { c:|rtvp |scenarios|slit.slf testout2.dat }
$$

Here, RTVP will start the raytracer and simulate both the measurement spectrum (taken with the instrument pointing to the center of the plume) and the reference spectrum. In this example, the reference spectrum is specified to have been taken pointing straight up (at an elevation angle of $90^{\circ}$ ).

Several result files are produced in the output folder. The files testout2.ref.txt and testout2.meas.txt are the simulated spectra, which can be viewed by using DOASIS. Notice that the results are still quite noisy if only 1,000 photons are used for each wavelength. The files testout2.refc.txt and testout2.measc.txt are the spectra after convolution with the slit function. Finally, the file testout2.od.txt is the simulated optical density, which can be compared with a specific measurement.

\section{Optical-Density at Specific Wavelengths Program Mode}

The Optical-Density at Specific wavelengths (ODS) program mode is similar to ODD mode; the only difference is that a list of wavelengths is additionally passed to RTVP in ODS mode. The program then calculates the optical density for these specific wavelengths. An example call of the ODS mode is.

rtvp -ods $300010007001 e 181090$ c:|rtvp|scenarios|slit.slf c:|rtvp|scenarios|calibration.clb testout2.dat

Here, the simulation will take place at the wavelengths corresponding to the individual spectrometer channels, as defined in the file calibration.clb. The output format is the same as in the ODD mode.

\section{Conclusions}

The DOAS method is increasingly being applied by volcanologists around the world to measure the emission rate of $\mathrm{SO}_{2}$ from volcanic vents. However, conventional DOAS retrievals fail to produce accurate results in situations where the measured volcanic plume is farther than about $2 \mathrm{~km}$ from the instrument, the plume contains high $\mathrm{SO}_{2}$ or significant aerosol loads, or if clouds or fog are present between the instrument and the plume (Kern and others, 2009).

In such cases, measurement accuracy can be significantly improved by utilizing an advanced retrieval technique such as the SRT-DOAS method (Kern and others, 2012). Though the principles of this method have already been described in great detail, the applicability of the technique by interested researchers has been limited by the complexity of the McArtim RTM. The software programs LTBuilder and RTVP address this issue. They provide a frontend to McArtim specifically for calculating lookup tables for use by the SRT-DOAS method and simulating UV radiative transfer in volcanic plumes. By using these tools, the SRT-DOAS approach should now be more readily accessible to scientists studying volcanic gas emissions with DOAS instruments. 


\section{Acknowledgments}

C. Kern would like to thank Mike Doukas, Chris Lockett, George Havach, and Jessica Dyke for their helpful reviews, which have greatly improved this report. Tim Deutschmann is gratefully acknowledged for providing the McArtim software and helpful advice on its use.

\section{References Cited}

Deutschmann, T., Beirle, S., Frieß, U., Grzegorski, M., Kern, C., Kritten, L., Platt, U., Prados-Román, C., Puksite, J., Wagner, T., Werner, B., and Pfeilsticker, K., 2011, The Monte Carlo atmospheric radiative transfer model McArtim-Introduction and validation of Jacobians and 3D features: Journal of Quantitative Spectroscopy and Radiative Transfer, v. 112, p. 1119-1137, doi: 10.1016/j.jqsrt.2010.12.009.

Kantzas, E.P., and Mcgonigle, A.J.S., 2008, Ground based ultraviolet remote sensing of volcanic gas plumes: Sensors, v. 8, p. 1559-1574.

Kern, C., Deutschmann, T., Vogel, L., Wöhrbach, M., Wagner, T., and Platt, U., 2009, Radiative transfer corrections for accurate spectroscopic measurements of volcanic gas emissions: Bulletin of Volcanology, v. 72, p. 233-247, doi: 10.1007/s00445-009-0313-7.

Kern, C., Deutschmann, T., Werner, C., Sutton, A.J., Elias, T., and Kelly, P.J., 2012, Improving the accuracy of $\mathrm{SO}_{2}$ column densities and emission rates obtained from upward-looking UVspectroscopic measurements of volcanic plumes by taking realistic radiative transfer into account: Journal of Geophysical Research, v. 117, no. D20, doi: 10.1029/2012JD017936.

Platt, U., and Stutz, J., 2008, Differential optical absorption spectroscopy; principles and applications: Springer-Verlag Berlin Heidelberg, 598 p. 